\title{
Enhance Fingerprint Image by Using Genetic Algorithms
} Ban Ahmed Mitras

dr.banah.mitras@gmail.com

College of Computer Sciences and Mathematics

University of Mosul

Received on: 20/04/2008

Daliya Abdullah

College of Education

University of Salah-Addin

Accepted on: 03/09/2008

\section{ABSTRACT}

In this paper, an algorithm is proposed to enhance fingerprint image which includes in one of its steps a smoothing process in which the suggested genetic algorithms by Mitras and Anwar in 2007 which used image smooth filters in both spatial and frequency domain will be employed to know their efficiency in enhancing and regaining the damaged sides of the fingerprint image to remove two types of noise, first one deals with noise added to the image, and the second one the noise already found in the image. Then histogram technique is used to enhance the fingerprint image.

Keyword: Genetic algorithm, image processing.

$$
\begin{aligned}
& \text { تحسين صورة بصمة الإصبع باستخدام خوارزميات جينية }
\end{aligned}
$$

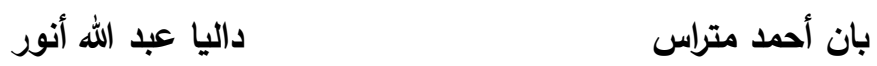

$$
\begin{aligned}
& \text { كلية علوم الحاسبات والرياضيات، جامعة الدوصل كلية التربية، جامعة صلاح الدين }
\end{aligned}
$$

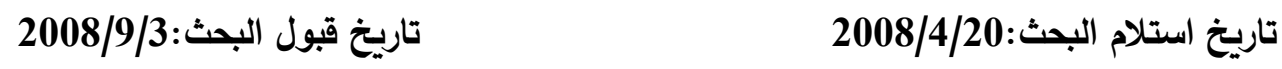

$$
\begin{aligned}
& \text { الملخص } \\
& \text { في هذا البحث تم اقتراح خوارزمية لتحسين صور بصمات الأصابع واستعادة حافات }
\end{aligned}
$$

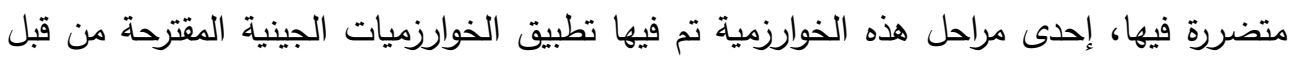

$$
\begin{aligned}
& \text { متراس وأنور في } 2007 \text { والتي تستخدم مرشحات تنعيم الصور المعروفة بأساليب مختلفة في }
\end{aligned}
$$

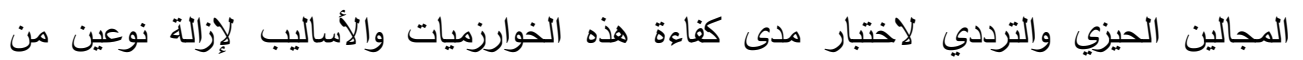

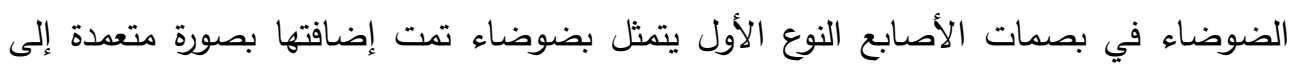

$$
\begin{aligned}
& \text { الصورة، أما النوع الثاني فهو يمثل ضوضاء موجودة بصورة مكتسبة، بالإضافة إلى تقنية تسوية } \\
& \text { المدرج التكراري. } \\
& \text { الكلمة المفتاحية: الخوارزمية الجينية ، معالجة الصوري، • مقدرية } \\
& \text { 1. Introduction مقدمة: } \\
& \text { استعمل البشر خصائص الجسم مثل الوجه والأذن والصوت للتعرف على بعضهم البعض ألمض }
\end{aligned}
$$

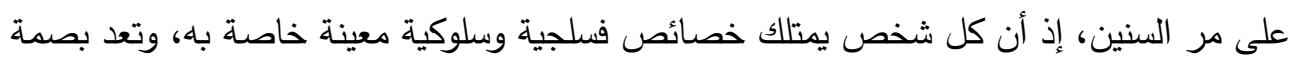

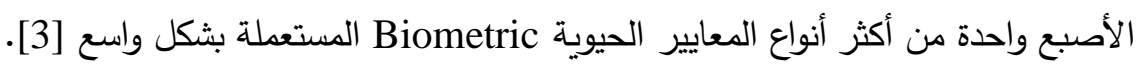


ويعتبر علم بصمة الأصبع كأحد العلوم الأكثر أهمية التي تحدد الأثخاص لأنه ليس

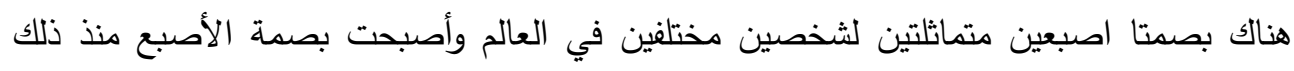
الوقت تستخدم على نطاق واسع للتحقيق في الجرائم [4].

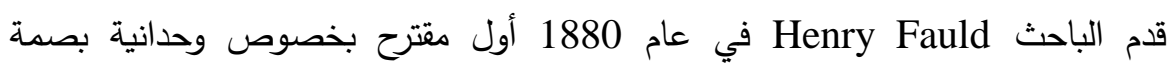
الأصبع • ثم أجرى Francis Galton (1912-1822) دراسة شاملة لبصمة الأصبع وقام بتعريف

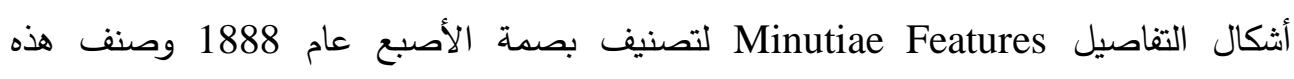
التفاصيل إلى ثلاثة أصناف رئيسة ( قوس Arch، دورة Loop، مستدير Whorl)

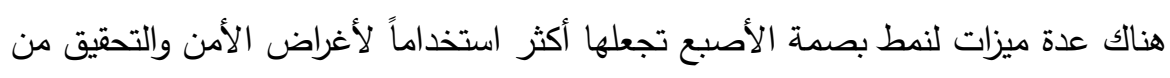

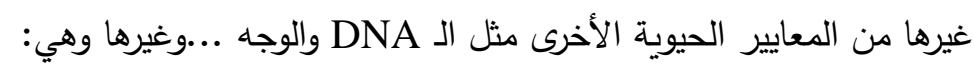

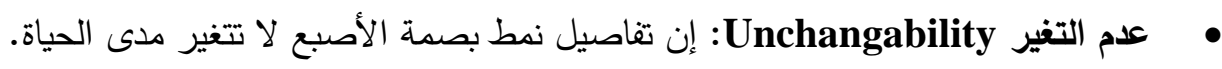

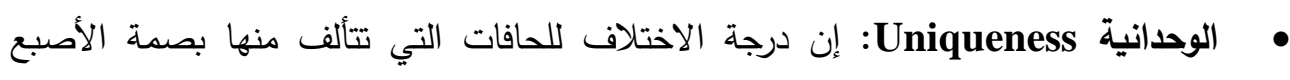

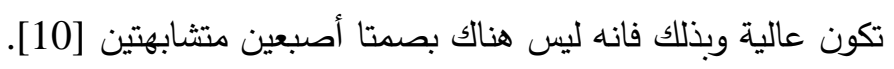

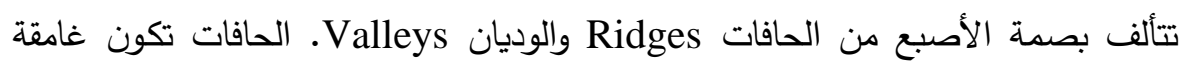

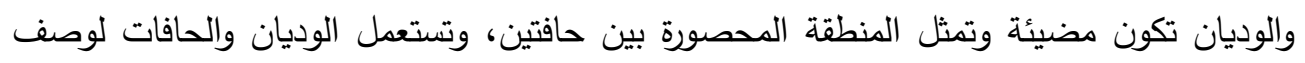
المناظر الطبيعية التي تكونها البصمة والتي تكون أحادية.

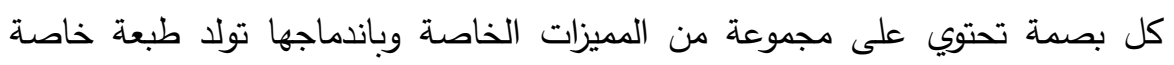

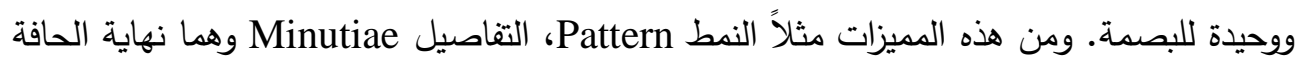

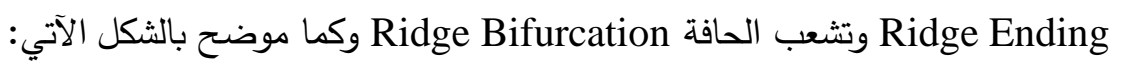

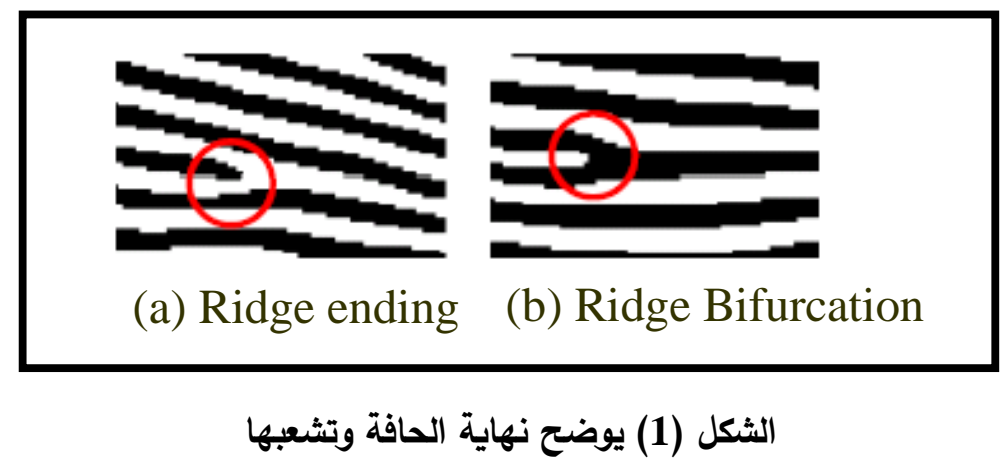

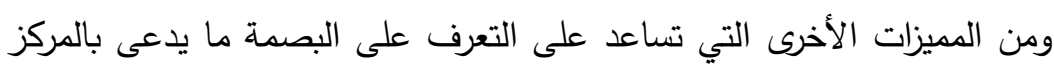

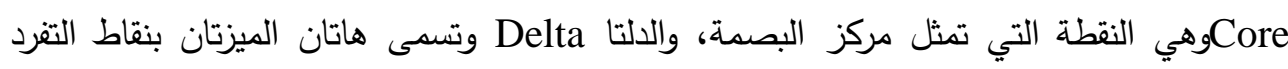
Singularity Points 


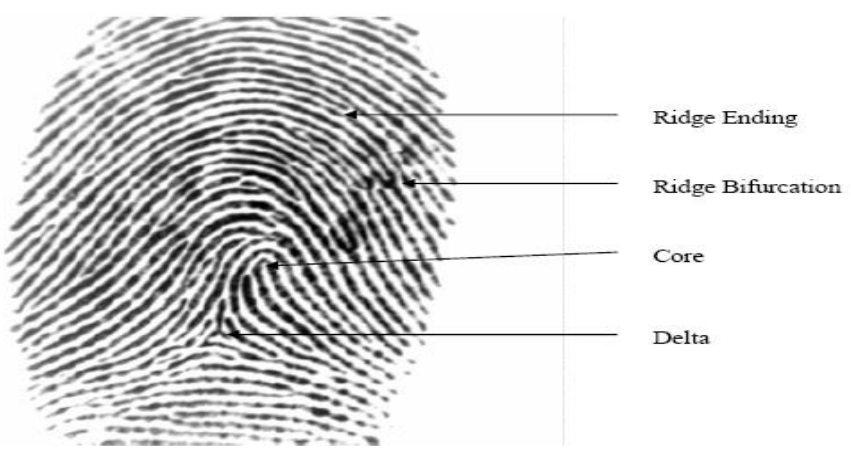

الثكل (2 ) يوضح نقطتي المركز Core والـ Delta

إن التقدم المهم في اكتثاف خصوصية بصمة الأصبع تعود لـ Edward Henry (1850-1931) والذي أسس ”Henry System”، لتصنيف بصمة الأصبع. وقام بتوسيع

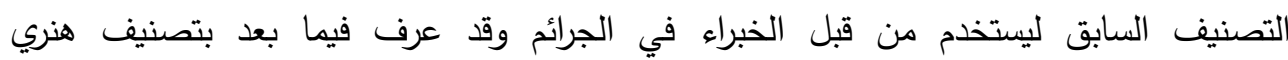
"Henry's Classification” 1. حلقات Loops: الحلقة من أكثر أنماط البصمة شيوعاً. وهذا النوع يتم تقسيمه دائماً إلى قسمين حلقات يمنى وحلقات يسرى. والاختلاف بينهما في الاتجاه فقط. 2. القوس Arch: وهو النمط الذي تكون فيه الخطوط التي تتألف منها الحافات تدخل من جانب التباه الطبعة Impression وترتفع في الوسط ثم تتدفق إلى خارج الجانب الأخر . 3. دوائر Whorls: في هذا النمط تنتشر الحافات بشكل دوائر متحدة المركز حول المركز . 4. مجموعات Combination: ممكن تجميع الأنواع المذكورة لتكوين نمط جديد، مثلا يوجد دمج دورج

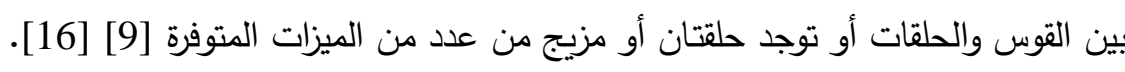

2. البحوث والدراسات السابقة Literature Review:

تعد خطوات تحسين صورة بصمة الأصبع من الخطوات الأساسية والمهمة في مجال

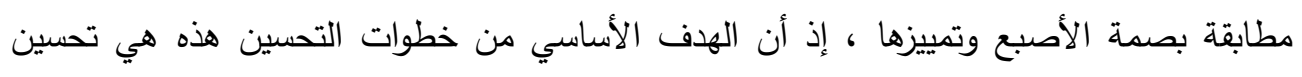

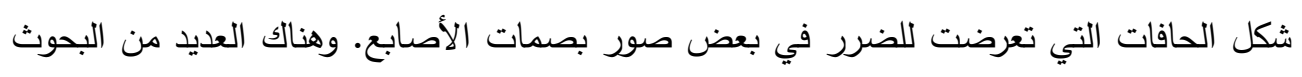
في مجال تحسين صورة بصمة الأصبع أهمها:

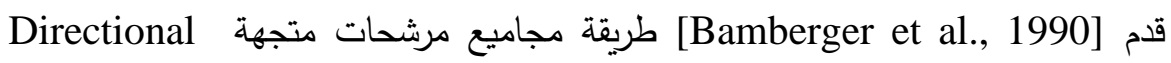

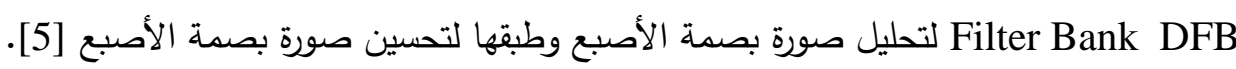
وقدم [Sherlock et al., 1994] طريقة لتحسين بصمة الأصبع معتمداً على تحويل

فورير ذي الوقت القصير Short Time Fourier Transform STFT [13. 
كما قدم [Hong, 1998] خوارزمية تحسين تعتمد على أجراء عملية اللف الرياضي

Ridge بالاعتماد على توجيه الحافة ألمحليه Convolution

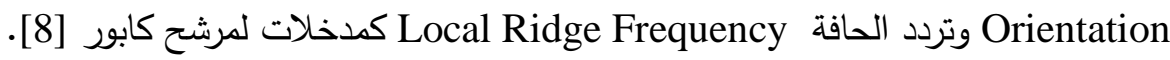
قدم [Greenberg et al., 2000] خوارزميتين لتحسين بصمة الأصبع الأولى والتي كالتي تعتمد على تعديل المدرج التكراري للصورة ثم استخدام مرشح وينر Wiener Filteringوبعدها يتم

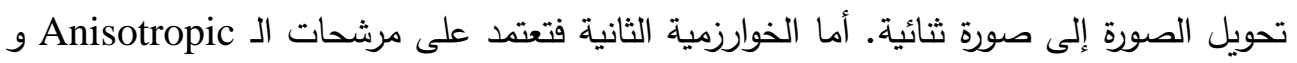
• لتكوين مرشح [7 Local Intensity Orientation كما قدم [Raymon, 2003] خوارزمية تعتمد على خوارزمية هونك ولكن بعد إضافة

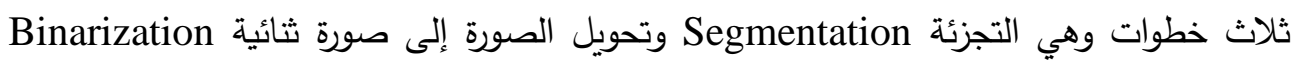

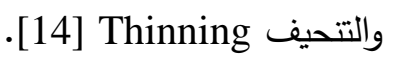
وقدم [Sang, 2003] طريقة لتحسين بصمة الأصبع بالاعتماد على مجاميع المرشحات الموجهه Directional Filter Bank (DFB) تعمل على تقليل تأثير الضوضاء على الحافات والوديان إذ تعمل على تصميم مرشح موجه ضد الضوضاء ينتشر في كل اتجاهات المجال الترددي

كما قدم [Wang et al.,2004] خوارزمية لتحسين صورة بصمة الأصبع تتكون من الخطوات الآتية: أولا إجراء عملية تعيير Normalization لقيم الانحراف القياسي والمعدل للصورة التي يتم معالجتها ثم تليها خطوة تطبيق مرشح كاوس لإزالة الضوضاء منها وأخيرا يتم تعديل المخطط البياني لها [15].

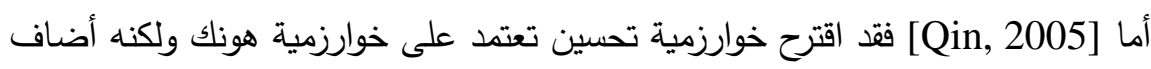

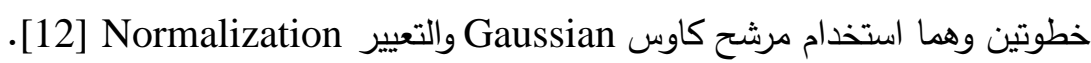
وأقترح [Cho et al.,2006] طريقة لتحسين صورة بصمة الأصبع باستخدام الخوارزمية

$$
\text { الجينية مع مرشح كابور [6]. }
$$

هناك العديد من خوارزميات التحسين لصورة بصمة الأصبع والتي تعتمد في إحدى مراحلها

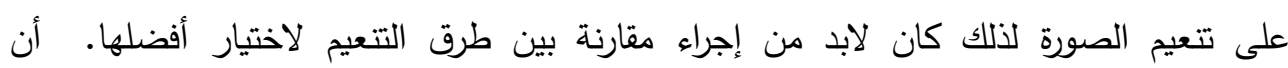

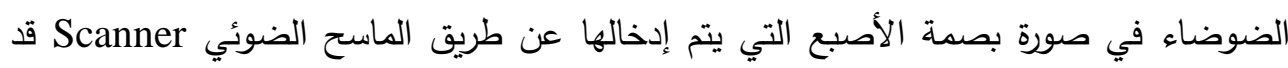
تكون ناتجة عن: 


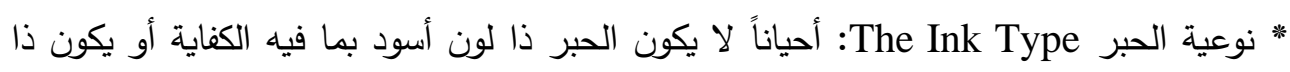

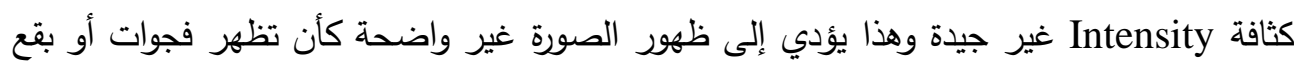
إضافية.

* قوة الضغط للطبع The Impression Force: إذا كانت البصمة قد تم الضغط عليها بعنف

على الورقة فأن العديد من البقع سوف تظهر على الصورة المأخوذة ضوئياً Scanned Image.

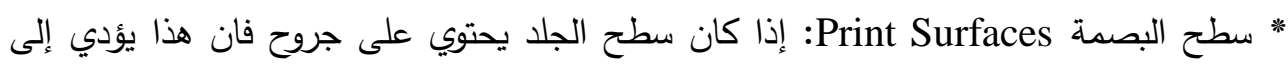

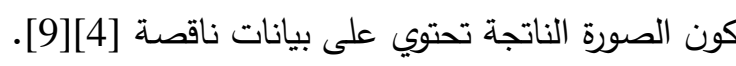

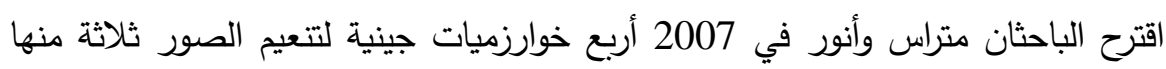
تعمل في المجال الحيزي والرابعة تعمل في المجال التون في الترددي فالخوارزمية الجينية الأولى تستخدم

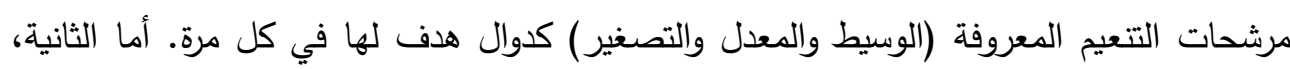

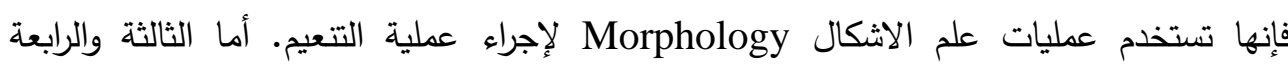

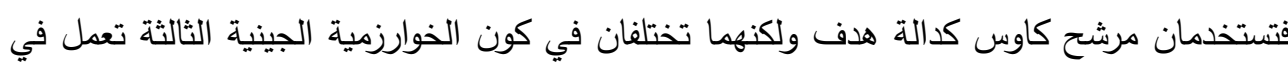
المجال الحيزي فيما تعمل الرابعة في المجال الترددي [2].

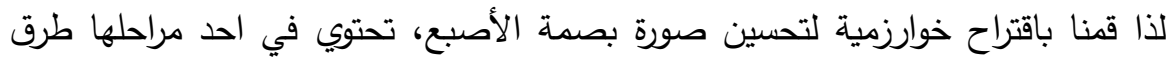

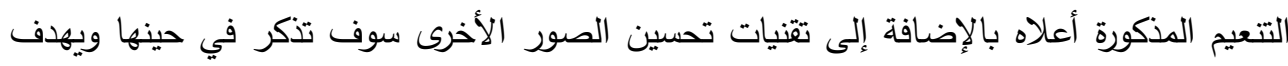

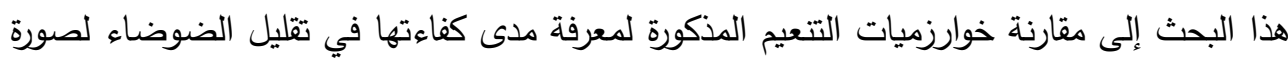
بصمة أصبع واستعادة أجزاء من الحافات المتضررة.

3. 3. الخوارزمية الجينية Genetic Algorithm

قام العالم جون هولاند John Holland وزملاؤه الخوارزمية الجينية عام 1975 في

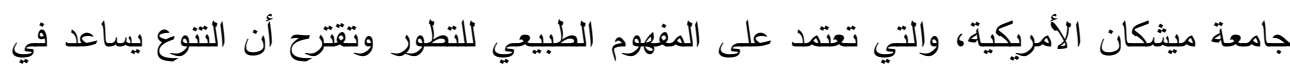

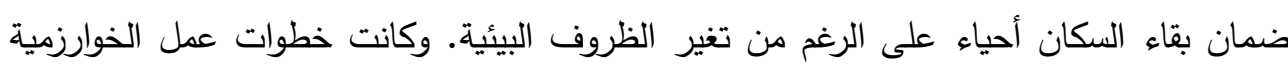

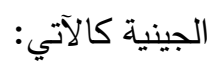
1 - [ البداية Start ] ] توليد جيل عشوائي يتكون من n من الكروموسومات.

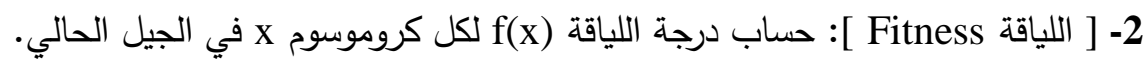

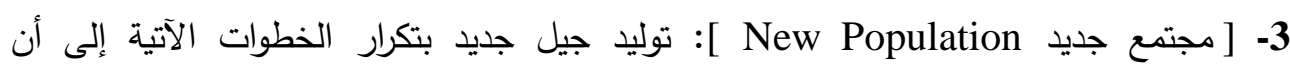

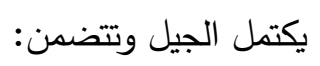

Parent Chromosome الاختيار Selection يتم اختيار اثثين من الكروموسومات التئل من الجيل حسب درجة اللياقة (أفضل القيم التي لها فرص أكبر للاختيار) . 
- التداخل الإبدالي Crossover: مع وجود احتمالية العبور أو التداخل الإبدالي بين الآباء المختارين لتوليد سلف جديد Children. - الطفرة Mutation: مع وجود احتمالية الطفرة، يتم عمل طفرة للسلف الجديد بموقع لونع

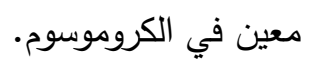
- الاستبدال Accepting: عملية وضع السلف الجديد المتكون في الجيل الجديد. - الاختبار Test: عند تحقق شرط التوقف، فإن الخوارزمية الجينية تتوقف، وتعيد الحل الجيد من آخر جيل متكون.

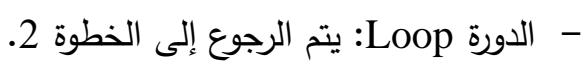

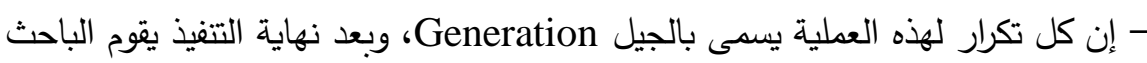

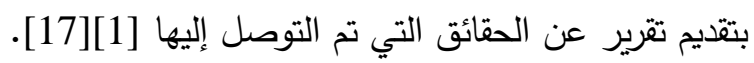
4. هيكلية عمل خوارزمية التحسين المقترحة:

نستعرض خطوات عمل خوارزمية التحسين المقترحة والتي تكون فيها الخطوة الثانية هي الخطوة الرئيسة وتتغير في كل طريقة تتعيم مستخدمة والخطوات هي: الخطوة الأولى: قراءة الصورة Image Read: يتم في البداية قراءة الصورة التي تتم معالجتها عن طريق الأمر (imread) وخزنها في مصفوفة معينة ولتكن

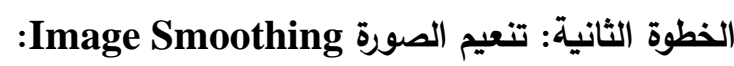

في هذه الخطوة يتم تطبيق الخوارزميات المقترحة في المصدر [2] وخزن الصورة الناتجة عن

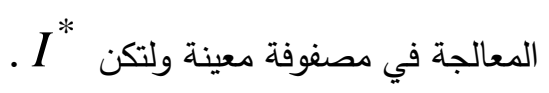
الخطوة الثالثة: في هذه الخطوة يتم طرح الصورة الأصلية التي تتم معالجتها I من الصورة الناتجة

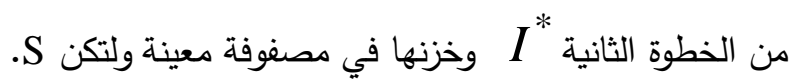
الخطوة الرابعة: مساواة المخطط البياني Histogram Equalizer: يتم في هذه الخطوة تحسين التباين في الصورة باستخدام تقنية مساواة المخطط البياني للصورة الناتجة عن عملية الطرح، وقد تم استخدام الدالة ( ) ل لتتفيذ هذه الخطوة برمجياً. 
الخطوة الخامسة: التحويل إلى صورة ثنائية Binarization: يتم تحويل الصورة التي تمت معالجتها في الخطوة (4) إلى صورة ثنائية وعرضها، وقد تم استخدام الدالة ( ) graythresh لتنفيذ هذه الخطوة برمجياً. والثكل (3) يبين خوارزمية التحسين المقترحة

المخطط الانسيابي للخوارزمية الجينية المقترحة

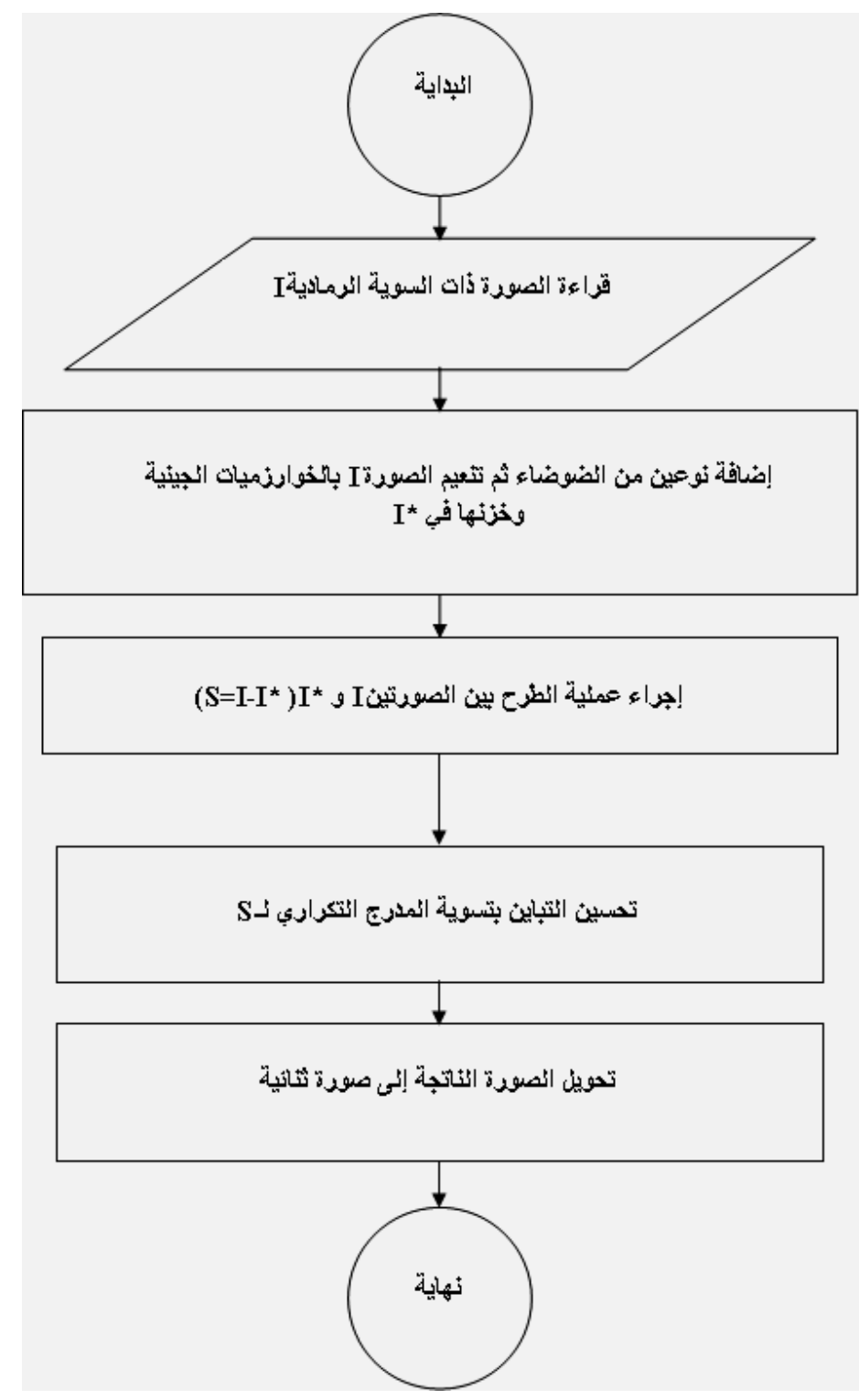

5. تطبيق خوارزمية التحسين المقترحة على صورة ذات نوعية غير جيدة: 
سوف نقوم بتطبيق الخوارزمية المقترحة على صورة بصمة اصبع ذات نوعية غير جيدة

تحتوي حافات متضررة. وفي الخطوة الثانية سنقوم بتطبيق الخوارزميات الجينية المقترحة في [2]. وسوف نقوم بعرض الصور في كل طريقة. والثكل (4) يوضح صورة البصمة التي تتم معالجتها والصورة بعد تحويلها إلى صورة ثنائية.

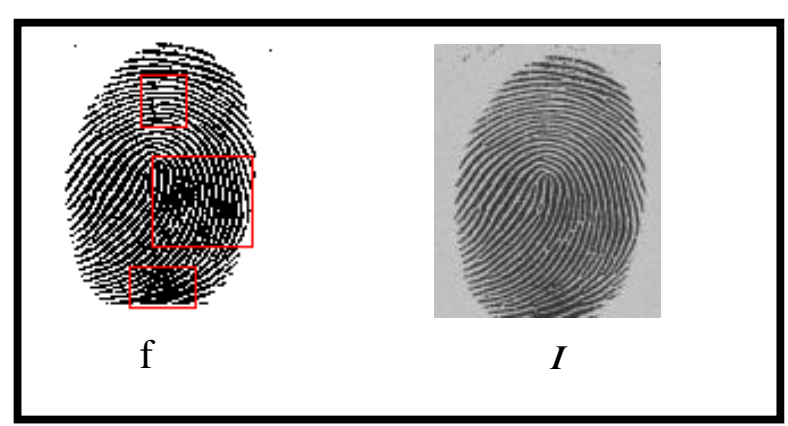

الثكل (4) الصورة I تمثل صورة البصمة التي سوف تتم معالجتها، الصورة تمثل الصورة I بعد تحويلها الى صورة ثنائية

5.1

بعد تطبيق خوارزمية التتعيم الجينية الأولى عندما كانت دالة الهدف من نوع الوسيط مع

خوارزمية التحسين المقترحة كانت الصور الناتجة بالثكل الآتي:

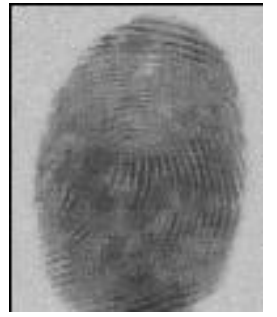

$\mathrm{I}^{*} 5 * 5$

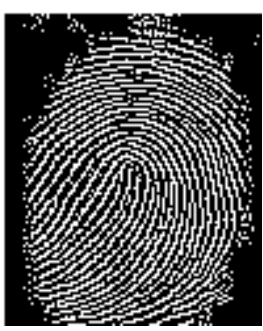

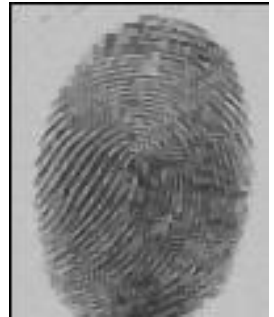

I $^{*} 3 * 3$

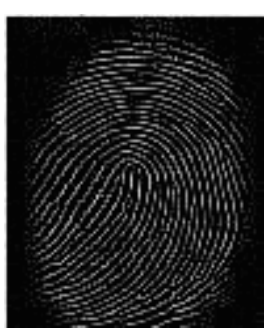

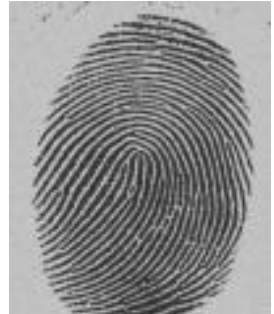

I

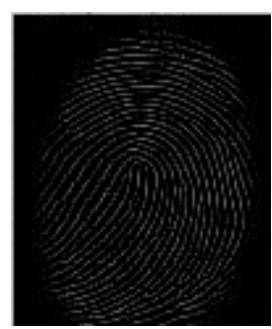


s3

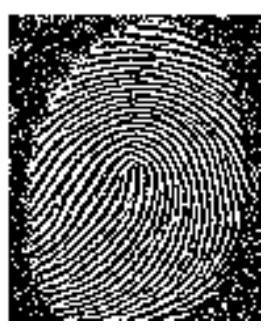

f3 s2

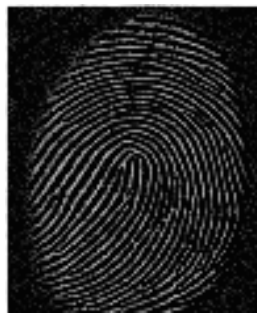

f2 s1

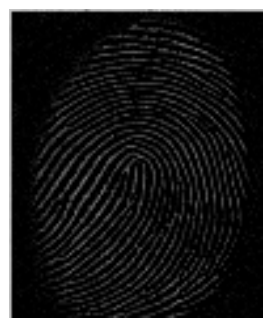

f1

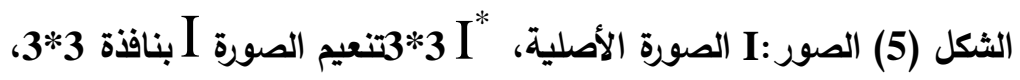

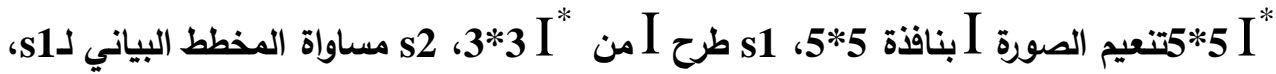

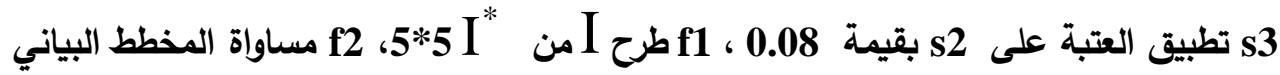

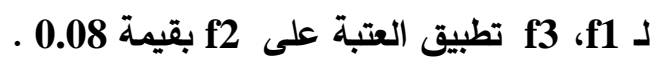

5.2 تطبيق خوارزمية التحسين المقترحة مع مرشح المعدل: بعد تطبيق خوارزمية التتعيم الجينية الأولى عندما كانت دالة الهدف من نوع المعدل مع خوارزمية التحسين المقترحة كانت الصور الناتجة بالثكل الآتي:

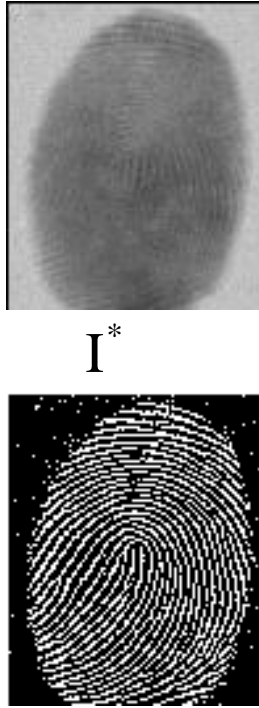

s3

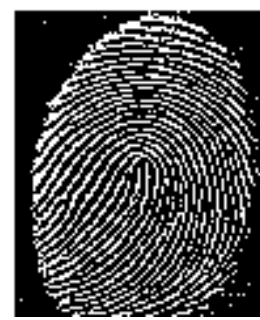

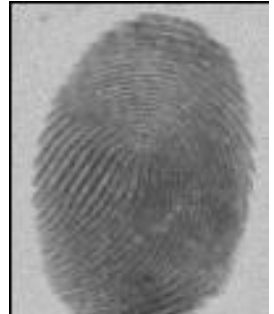
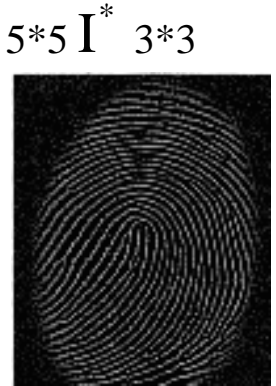

s2

65

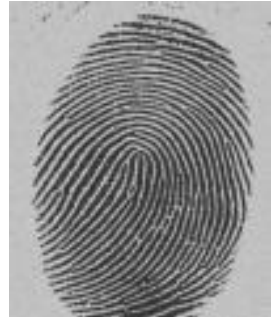

I

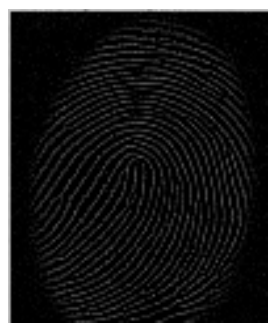

s1 
بان أحد حسن ودالثيا عبد الله أنور

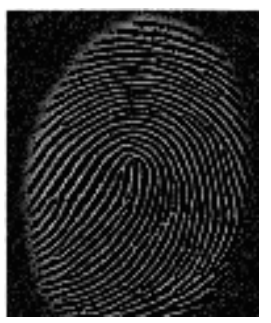

f2

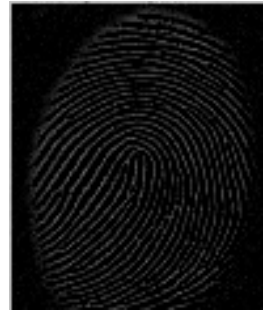

f1

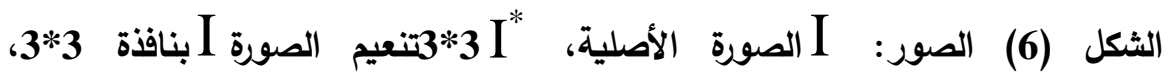

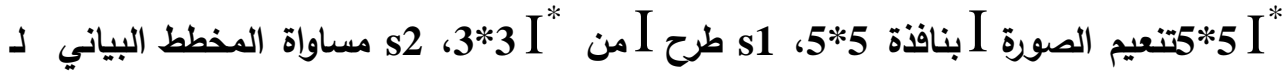

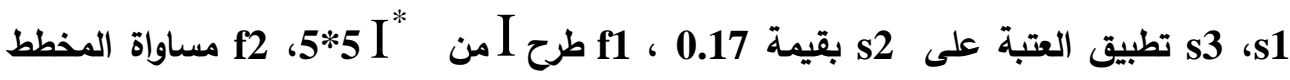

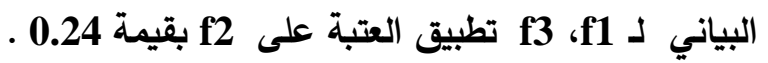
5.3 تطبيق خوارزمية التحسين المقترحة مع مرشح التصغير: بعد تطبيق خوارزمية التتعيم الجينية الأولى عندما كانت دالة الهدف من نوع مرشح التصغير مع خوارزمية التحسين المقترحة كانت الصور الناتجة بالثكل الآتي:
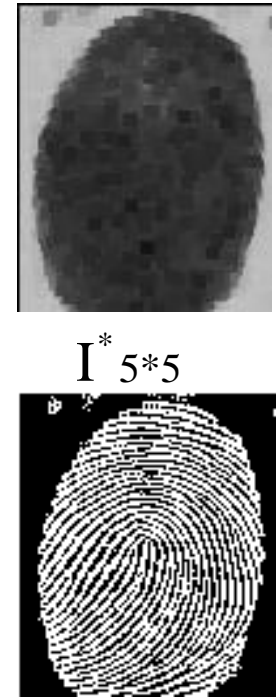

s3

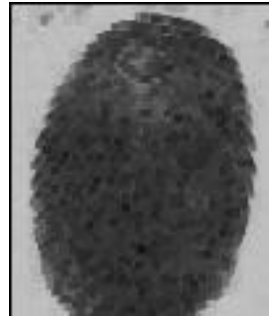

$I^{*} 3 * 3$

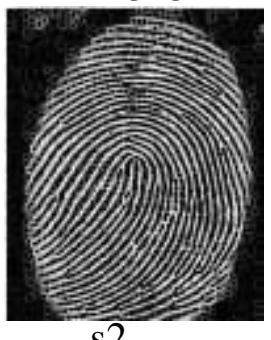

s2 66
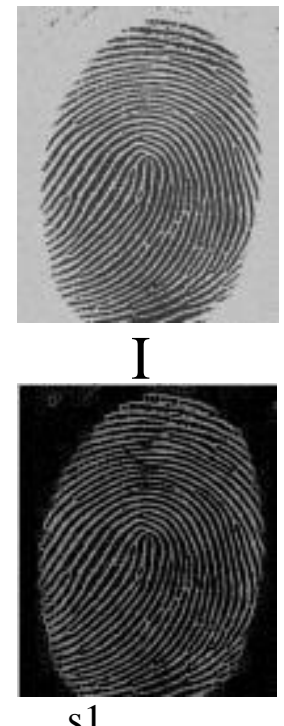

s1

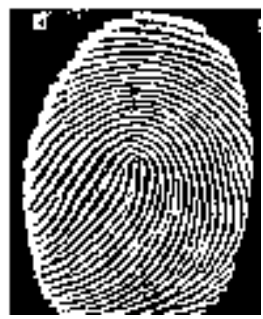




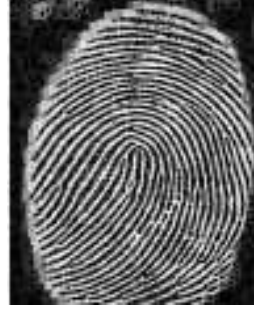

f2

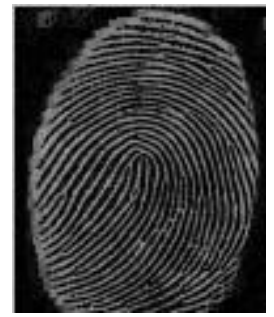

f1

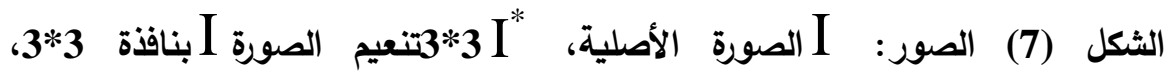

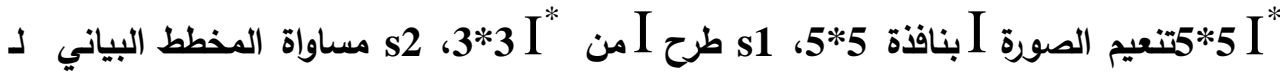

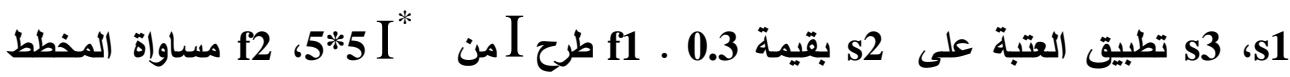

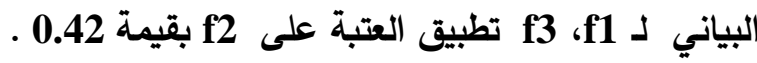
5.4 بعد تطبيق خوارزمية التتعيم الجينية الثانية مع خوارزمية التحسين المقترحة كانت الصور

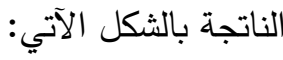

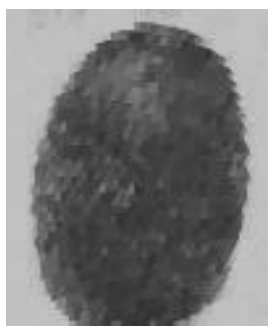

f2

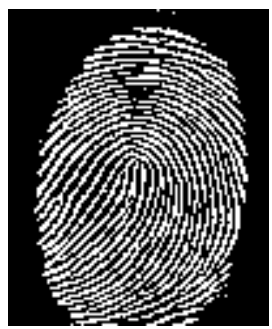

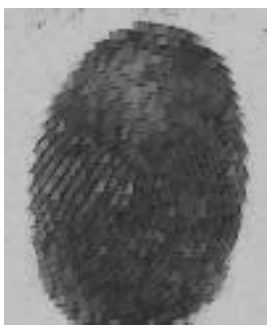

f1

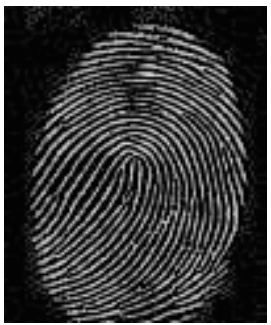

67

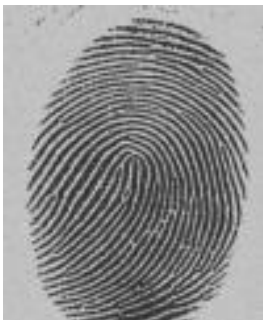

I

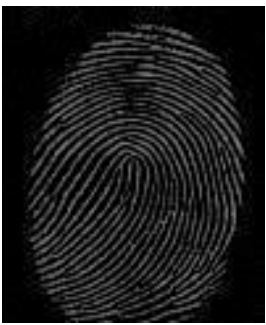


بان أحمد حسن وداليا عبل الله أنور

s3

s2

s1

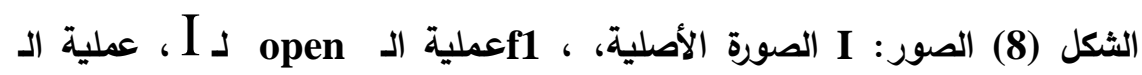

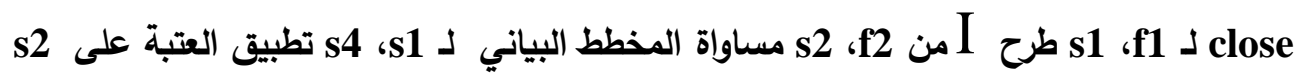

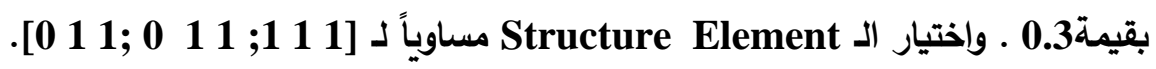

5.5 تطبيق خوارزمية التحسين المقترحة مع مرشح دالة كاوس في المجال الحيزي:

بعد تطبيق نتائج خوارزمية التتعيم الجينية الثالثة مع خوارزمية التحسين المقترحة كانت

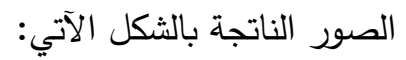
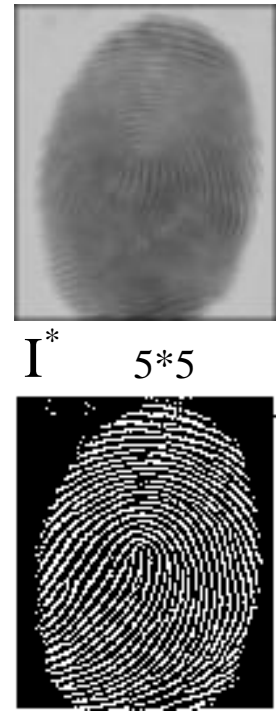

s3

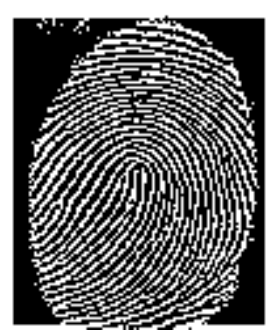

f3

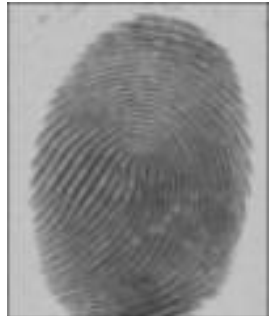

$3 * 3$

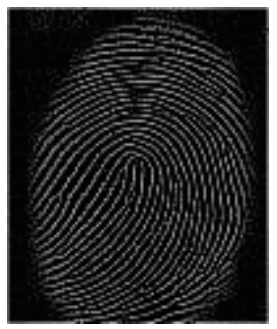

s2

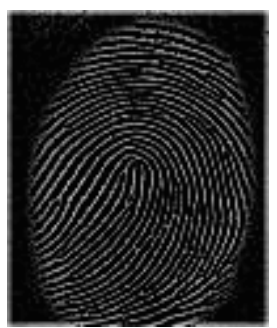

f2

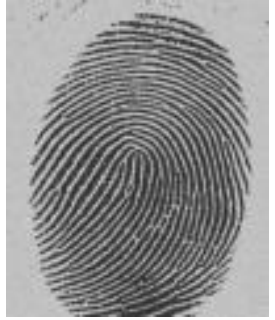

I

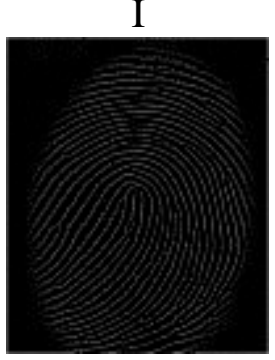

s1

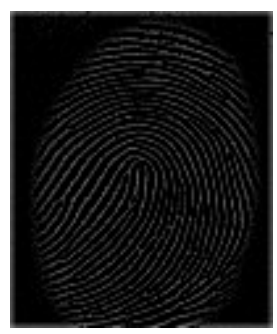

f1 


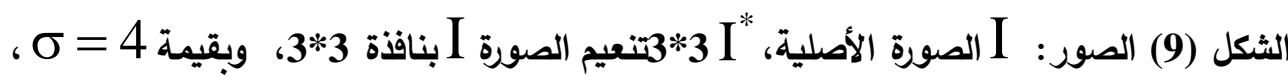

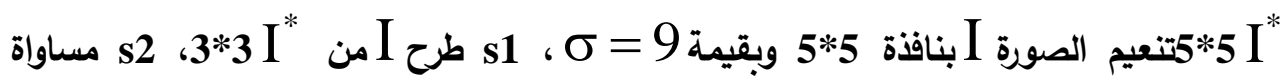

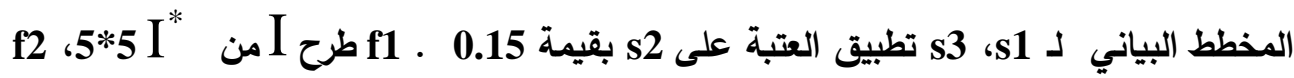

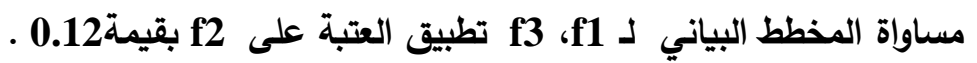
5.6 تطبيق خوارزمية التحسين المقترحة مع مرشح دالة كاوس في المجال الترددي: بعد تطبيق خوارزمية التتعيم الجينية الرابعة مع خوارزمية التحسين المتترحة كانت الصور

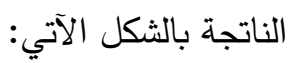

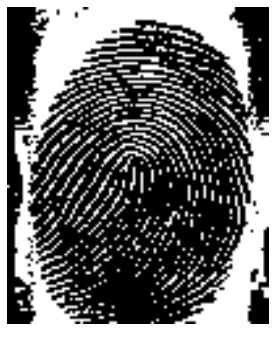

$\mathrm{f}$

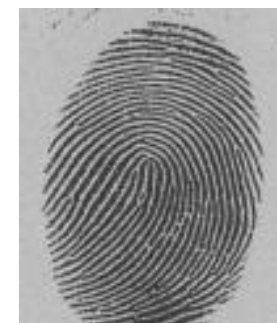

$\mathrm{S}$

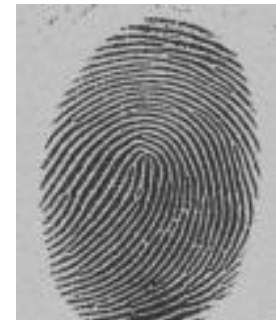

I

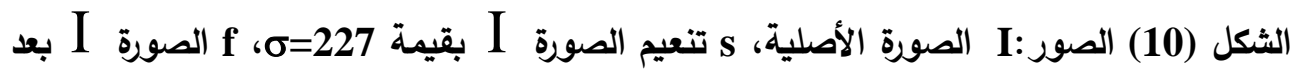
تطبيق خوارزمية التحسين المقترحة وبقيمة عتبة تساوي 0.035. ولمعرفة تأثير خوارزميات التتعيم المتترحة في [2] على صورة البصمة التي تتم معالجتها فقد قمنا

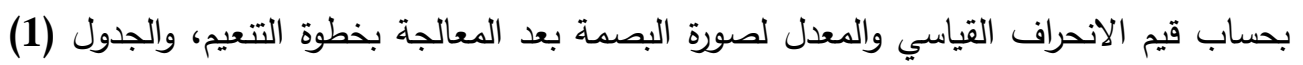
يبين هذه القيم، وإن قيمة الانحراف القياسي لصورة البصمة قبل المعالجة تساوي (51.7798) والمعدل يساوي (140.1959). جدول(1) يمثل قيم الانحراف القياسي والمعدل لصورة البصمة بعد تطبيق خوارزميات التنعيم

\begin{tabular}{|c|c|c|c|}
\hline 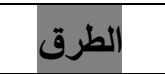 & حجم النافذة حجة & Std. الاحمراف القياسي & المعدل Mean \\
\hline \multirow{2}{*}{ Median } & $3 * 3$ & 46.9338 & 135.0158 \\
\hline & $5 * 5$ & 45.7234 & 133.7873 \\
\hline \multirow[t]{2}{*}{ Mean } & $3 * 3$ & 43.3568 & 136.0156 \\
\hline & $5 * 5$ & 41.8660 & 134.3248 \\
\hline \multirow[t]{2}{*}{ Min } & $3 * 3$ & 60.4306 & 96.2066 \\
\hline & $5 * 5$ & 48.0362 & 84.1573 \\
\hline
\end{tabular}


بان أحدد حسن وداليا عب الله أنور

\begin{tabular}{|c|c|c|c|}
\hline Gaussian 1 & $\mathbf{3} * 3$ & $\mathbf{3 8 . 7 8 5 5}$ & 137.9859 \\
\cline { 2 - 4 } & $\mathbf{5} * \mathbf{5}$ & $\mathbf{3 7 . 0 5 6 6}$ & $\mathbf{1 3 6 . 3 1 0 7}$ \\
\hline Gaussian 2 & $\ldots .$. & $\mathbf{4 8 . 3 5 1 4}$ & 140.1941 \\
\hline Morphology & $\mathbf{3} * 3$ & $\mathbf{5 6 . 4 6 9 5}$ & 116.0828 \\
\hline
\end{tabular}

وبملاحظة النتائج الموضحة في الجدول السابق نجد أن الخوارزمية الجينية الأولى عندما كانت دالة الهدف طريقة المعدل المحسنة نجدت في تقليل قيم الانحراف القياسي والمعدل لصورة البصمة وتلتها الخوارزمية الجينية الثالثة ثم الخوارزمية الجينية الأولى عندما كانت دالة الهئه الهدف

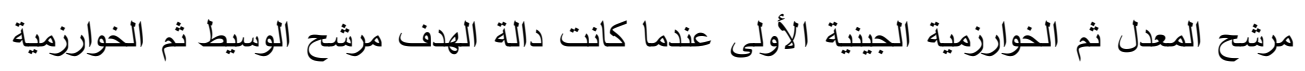

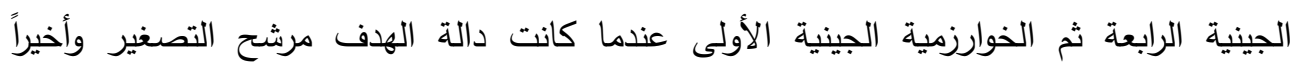
الخوارزمية الجينية الثانية.

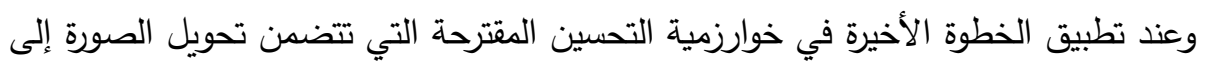
صورة ثنائية فقد تم استخدام طريقة العتبة لتحويل الصورة وان أفضل قيم العتبة لخوارزمية التحسين المقترحة موضحة في الجدول الآتي: جدول (2) أفضل العتبات المستخدمة

\begin{tabular}{|c|c|c|}
\hline الطرق ل & حجم الناقذة & قيمة العتبة \\
\hline \multirow{2}{*}{ Median } & $3 * 3$ & 0.08 \\
\hline & $5 * 5$ & 0.08 \\
\hline \multirow{2}{*}{ Mean } & $3 * 3$ & 0.17 \\
\hline & $5 * 5$ & 0.24 \\
\hline \multirow{2}{*}{ Min } & $3 * 3$ & 0.3 \\
\hline & $5 * 5$ & 0.42 \\
\hline \multirow{2}{*}{ Gaussian 1} & $3 * 3$ & 0.15 \\
\hline & $5 * 5$ & 0.12 \\
\hline Gaussian 2 & $\ldots .$. & 0.035 \\
\hline Morphology & $3 * 3$ & 0.3 \\
\hline
\end{tabular}

من هذا الجدول يتبين أن أفضل قيم للعتبة كانت محصورة بين (0.45-0.03).

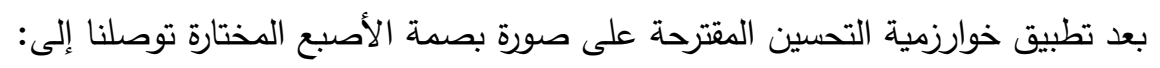

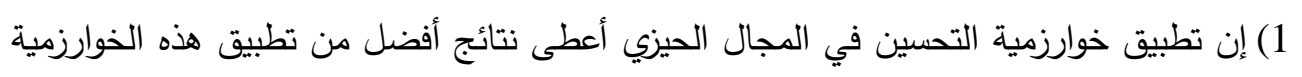

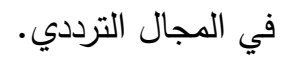


2) إن تطبيق خوارزمية التحسين مع خوارزميات التتعيم المقترحة في المجال الحيزي نجحت معظمها في تخمين الحافات المتضررة واستعادتها. 3) إن قيمة العتبة المستخدمة لتحويل الصورة إلى صورة ثنائية كان لها تأثير كبير في إبراز الكثير من التفاصيل في الصورة وإخفائها.

4) إن أفضل نتيجة تم الحصول عليها كانت بتطبيق خوارزمية التحسين مع الخوارزمية الجينية

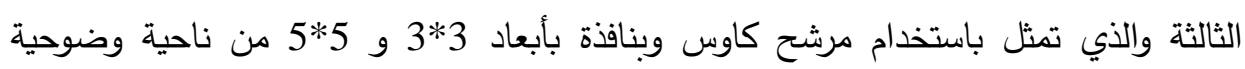

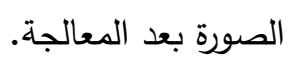


بان أحدد حسن ودالثيا عب النه أنور

$$
\begin{aligned}
& \text { المصادر } \\
& \text { بشير، غصون سالم. (2003)، " استخدام الخوارزمية الجينية في مطابقة الصور"، } \\
& \text { بحث ماجستير، جامعة الموصل، كلية علوم الحاسبات والرياضيات،علوم الحاسبات. } \\
& \text { متراس، بان احمد و أنور، داليا عبد الله, (2007)، " خوارزميات جينية كفوءة لتتعيم } \\
& \text { الصور" ، المجلة العراقية للعلوم الإحصائية. العدد 1802. }
\end{aligned}
$$

[3] Agarwal, M. (2006), "Multi-Impression Enhancement of Fingerprint Images", M.Sc., Thesis, West Virginia University, College of Engineering and Mineral Resources.

[4] AL-Wattar, T.Y.H (2000), "Fingerprints Recognition Using Neural Networks", M.sc. Thesis, University of Mosul.

[5] Bamberger, R. H. (1990), "The Directional Filter Bank: A Multirate Filter Bank for the Directional Decomposition of Images", ph.D. Theses, Georgia Institute of Technology.

[6] Cho, U.; Hong, J. and Cho, S. (2006), "Evolutionary singularity Filter Bank Optimization for Fingerprint Image Enhancement", LNCS 3907, pp.380-390.

[7] Greenberg, S.; Aladjem, M.; Kogan, D. and Dimitrov, I. (2000), "Fingerprint Image Enhancement Using Filtering Techniques", In: Proc. 15th Internat. Conf. on Pattern Recognition III, Barcelona, Spain, pp. 326-329.

[8] Hong, L.; Wan, Y. and Jain, A. (1998), "Fingerprint Image Enhancement Algorithm and Performance Evaluation", IEEE Trans. Anal. Machine Intell, Vol. 20, No. 8, pp. 777-789.

[9] Jain, A. K.; Hong, L. and Prabahakar, S. (1999), “A Multichannel Approach to Fingerprint Classification", IEEE Trans. On Pattren Analysis and Machine. Intelligence, Vol. 21, No. 4, pp. 348-359.

[10] Lee, H. C. and Gaensslen, R. E. (1991), "Advances in Fingerprint Technology", Elsevier, New York.

[11] Oh, S. K.; Lee, J. J.; Park, C. H.; Kim, B. S. and Park, K. (2003), "New Fingerprint Image Enhancement Using Directional Filter Bank", Journal of WSCG, Vol.11, No.1., ISSN 1213-6972. 
[12] Qin, M. (2005), "A Fast and Low Cost SIMD Architecture for Fingerprint Image Enhancement", M.Sc., Thesis, Faculty of Electrical Engineering, Mathematics and Computer Science, Department of Electrical Engineering.

[13] Sherlock, B.G.; Monro, D.M. and Millard, K. (1994), "Fingerprint Enhancement by Directional Fourier Filtering”, IEE Proc. Vision Image Signal Process, Vol. 141, No. 2, pp 87-94.

[14] Thai, R. (2003), "Fingerprint Image Enhancement and Minutiae Extraction", The University of Western Australia.

[15] Wang, S., and Yangsheng W. (2004), "Fingerprint Enhancement in the Singular Point Area", IEEE Signalprocessing Letters, Vol. 11, No. 1, January.

[16] Ganapathi, S.k., (2002), "Fingerprint Authentication:Shifting The Electronic Security Paradigm", SC Magazine,January 29. http://www.scmagazine.com/sconline.htm.

[17] Marczyk, A. (2004), "Genetic Algorithms and Evolutionary Computation",April,Vol.23.

http://www.talkor:gins.org/Faqs/genalg/genalg.html/\#introductin 